\title{
Pulsatile Blood Flow in Anatomically Accurate Vessels with Multiple Aneurysms: A Medical Intervention Planning Application of Computational Haemodynamics
}

\author{
IORDANIS CHATZIPRODROMOU ${ }^{1}$, VINCENT D. BUTTY ${ }^{1}$, \\ VINOD B. MAKHIJANI ${ }^{2}$, DIMOS POULIKAKOS ${ }^{1}$ and \\ YIANNIS VENTIKOS ${ }^{1, \star}$ \\ ${ }^{1}$ Laboratory for Thermodynamics in Emerging Technologies, Department of Mechanical and \\ Process Engineering, Swiss Federal Institute of Technology, CH-8092 Zurich, Switzerland \\ ${ }^{2}$ CFD Research Corporation, Biomedical Technology Branch, Huntsville, AL, U.S.A.
}

Received 8 October 2002; accepted in revised form 8 June 2003

\begin{abstract}
In the present study, we are investigating computationally the pulsatile flow in an anatomically accurate cerebral arterial segment exhibiting two saccular aneurysms. Our focus is on the haemodynamic patterns observed within the two aneurysms, in terms of inflow-outflow regions, emergence and disappearance of coherent structures and mixing throughout the cardiac cycle. The results obtained carry interesting features, important for thrombosis, pharmacokinetics and particularly for interventional planning for aneurysm treatment. For the latter, being the center-point of this study, we show that the two aneurysms behave in a dissimilar manner, since the blood inflow region oscillates significantly for one of them and practically does not oscillate at all for the second. This information can guide the medical interventionist in designing the optimal approach, particularly in cases where total obliteration of the aneurysm neck opening is impossible.
\end{abstract}

Key words: blood flow, cerebral aneurysm, computational haemodynamics, pulsatility.

\section{Introduction}

Cerebral saccular aneurysms are balloon-like deformations of the arterial wall of the vessels that bring blood to the brain (Figure 1). The rupture of aneurysms of this type often has particularly harmful consequences, since it leads to hemorrhage in the subarachnoidal region and accounts for almost $7 \%$ of all strokes. Moreover, approximately $30 \%$ of all aneurysm ruptures are lethal immediately, and an additional $40 \%$ lead to death within 4 weeks, if the rupture is not treated effectively. The sheer number of studies of this nature done over the last 30 years $[1,2,7,8,11,16$, $17,20]$, only underlines the importance of such demographics, importance which is

\footnotetext{
^ Author for correspondence. Currently at Department of Engineering Science, University of Oxford, Parks Road, Oxford OX1 3PJ, U.K.; E-mail: yiannis.ventikos@engineeringscience.oxford.ac.uk.
} 


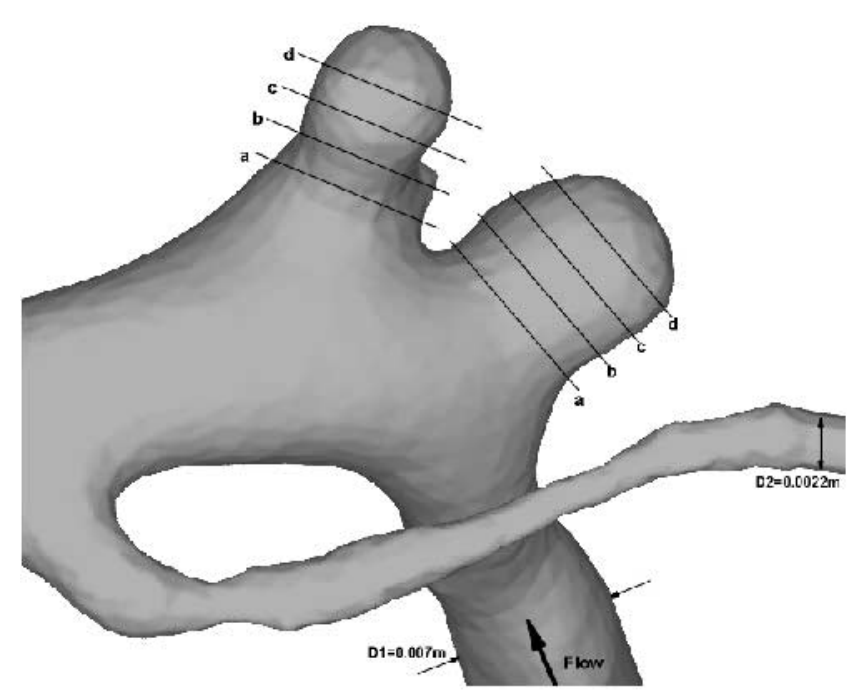

Figure 1. Domain of interest and control cross-sections through the two aneurysms. (Original in colour)

further reinforced by the fact that, at least according to some estimates, $1-5 \%$ of the general population exhibits cerebral aneurysms. The above figures do not include the side-impact that cerebral aneurismal sacs may have in thrombus creation, since the recirculating, sometimes almost stagnant, blood inside the aneurysm presents with an increased potential for clotting [15].

Computational Fluid Dynamics, when combined with proper insight on the physiology of the human body system under investigation, can provide us with valuable information concerning both the mechanisms at play and the planning of mission-critical medical interventions. Computational simulation of aneurismal flows has already allowed for a substantial increase of our understanding of the prevailing haemodynamics $[3,9,13]$. Visual inspection of the simulated flow patterns allows for valuable insight on the blood behavior near the orifice, or neck, of the aneurysm, but also within the sac. Moreover, the use of haemodynamic analysis also allows for quantities like strain and wall shear stress to be mapped onto the vascular surface. This may contribute to better planning of endovascular treatment, and to our understanding of aneurysm rupture risk and patterns.

When numerical tools involving the solution of the Navier-Stokes equations are to be utilized routinely within a clinical environment, sufficient preparatory work has to be conducted in order to pre-establish all the parameters and requirements in a concrete way that covers all the possible situations and configurations the interventionist might encounter. This element, being of critical importance when designing an intervention planning decision support methodology, is partly driving the present effort. 


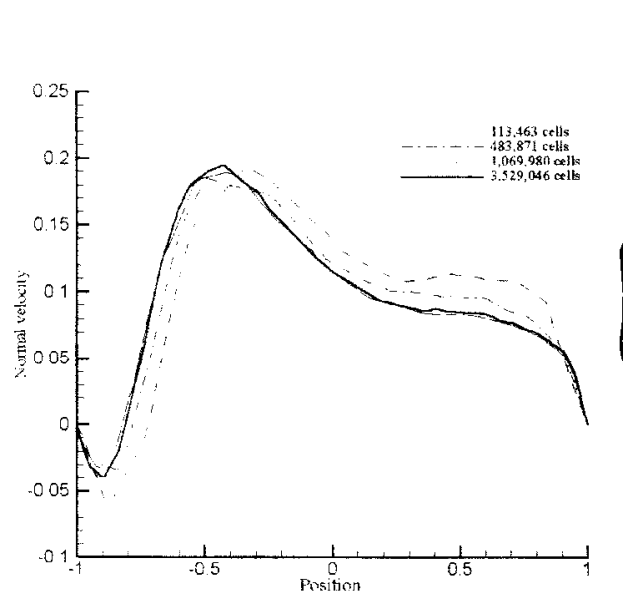

(a)

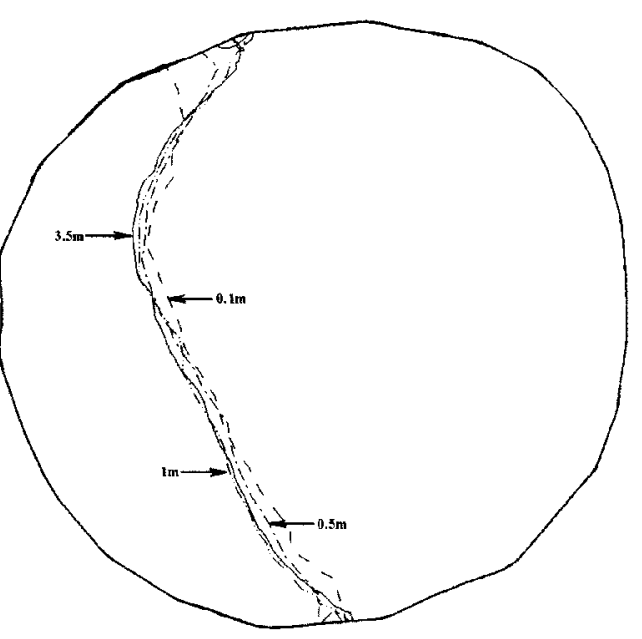

(b)

Figure 2. Grid independence study: (a) Influence of mesh resolution on the computation of the normal velocity profiles along a line on the cross-sectional plane (a) at the neck of the first aneurysm. (b) Influence of mesh resolution on the computation of the inflow/outflow regions separating line (zero normal velocity contour line) on the cross-sectional plane (a) at the neck of the first aneurysm.

We shall present results from a computational simulation of the pulsatile flow within a patient's right internal carotid artery exhibiting two saccular aneurysms very close to each other. It is often the case that such aneurysms are obliterated through coil embolization, i.e. through the introduction (via a catheter) of a thin platinum wire of adequate length, within the aneurysm. This coil forms a densely packed grid and acts as a porous medium, thus diminishing or eliminating completely the blood flow within the aneurysm and leading to the formation of a stable thrombus. Both the desired location of coil insertion, but also the thrombotic features of any particular aneurysm depend strongly on its haemodynamics, as they are governed by the pulsatile blood flow characteristics and the geometric specifics of the sac and parent vessel.

\section{Procedure and Numerical Techniques}

There are a number of steps that have to be completed when patient-specific haemodynamic computations are to be performed. The procedure, briefly, has to go through several stages [3], including:

- The acquisition of a suitable set of medical images that adequately describe the region of interest. These might be Computer Tomography data, Magnetic Resonance Imaging data, 3D Angiography data, etc. 


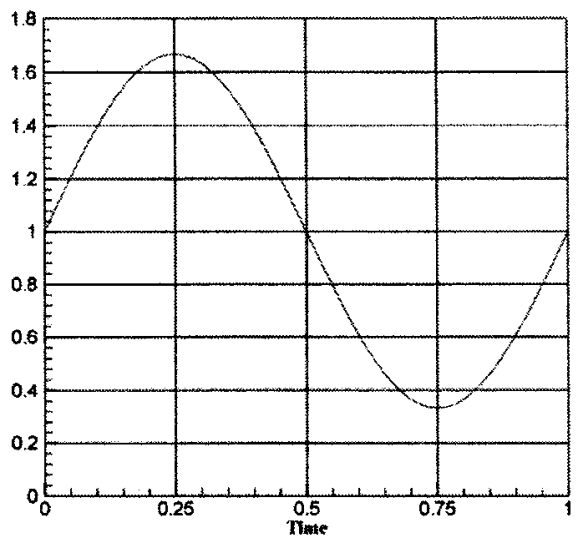

Figure 3. Sinusoidal waveform of the inflow boundary condition.

- The processing of the images to extract the geometric features of the region of interest.

- The discretization of the volume enclosed within the extracted surfaces with a suitable mesh generation technique.

- The numerical solution of the pertinent equations for the blood motion.

In the present study, we are utilizing CT data for a subject with two saccular aneurysms at the supraclinoid portion of the right internal carotid artery. The image stack used has a resolution of $512 \times 512$ pixels at 74 slices with a pitch of $0.5 \mathrm{~mm}$ $\times 0.5 \mathrm{~mm} \times 1 \mathrm{~mm}$ and was acquired using a Toshiba Helical CT scanner [6]. The images are processed [5, 18], and the extracted surface geometry is imported in a grid generator, (Hypermesh by Altair Eng.), where an unstructured, triangular surface description and a volume tetrahedral-based grid are created.

Subsequently, the flow of blood through this geometry is obtained. For the numerical solution of the flow in question, the CFD-ACE solver, by CFD Research Corporation is used. This solver employs a finite volume approach on a fully unstructured geometry for the solution of the incompressible, unsteady, three-dimensional Navier-Stokes equations, allowing for the incorporation of nonNewtonian fluid models, as well as for interaction of the fluid with the solid wall $[10,19]$. Algebraic multigrid acceleration was employed in all simulations performed in order to enhance the speed of convergence of the solution.

A significant number of steady and pulsatile flow computations, with a variety of numerical parameters, has been performed in order to eliminate, as much as possible, the influence of numerics on the solution. For this reason, computations with grids ranging from approximately 100,000 to 3,500,000 elements and several numerical discretization schemes were conducted. We should reiterate at this point that for all practical purposes, i.e. for the application of computational haemodynamics in a clinical environment, very strict quality criteria in terms of grid dependence and numerical sensitivity have to be pre-established. This means that 


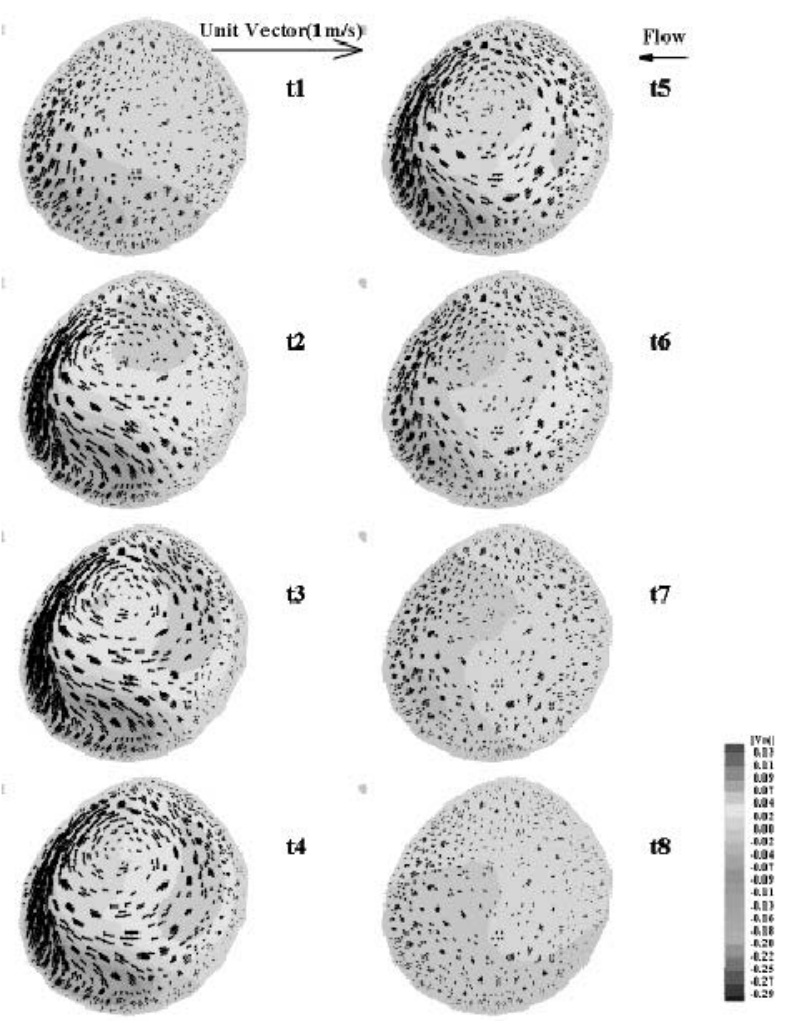

Figure 4a. Evolution of the inflow-outflow patterns and cross-flow vectors for the cross-section 'a' of the first aneurysm. (Original in colour)

for every possible patient geometry, the requirements for an accurate solution must be known a priori, since neither the time nor the resources are usually available for a detailed study in each clinical case. In effect, the grid independence analysis of this and similar geometries, critical for establishing such rules and guidelines, is a topic of paramount importance that has begun attracting attention recently [14]. Therefore, we shall present a detailed investigation defining a numerical accuracy protocol for a critical cerebral pathology in a different publication [4]. For completeness however, we are briefly presenting an indicative grid independence study for a quantity of great interest to this work, i.e. the normal velocity at the neck of one of the aneurysms. Figure 2a depicts this normal velocity along a line on the cross-sectional plane (a) at the neck of the first aneurysm (Figure 1). It is apparent, that for this velocity all grids but the coarsest one yield similar results. The error in maximum positive and negative velocity, normalized with the local value, is less than $4 \%$, when our reference grid (the one with approximately half a million cells) and the finest grid are compared. Figure $2 b$ shows an even more convincing trend: the lines depicted correspond to the contour of zero normal velocity, thus delineating the regions of inflow and outflow for this particular aneurysm (for the 

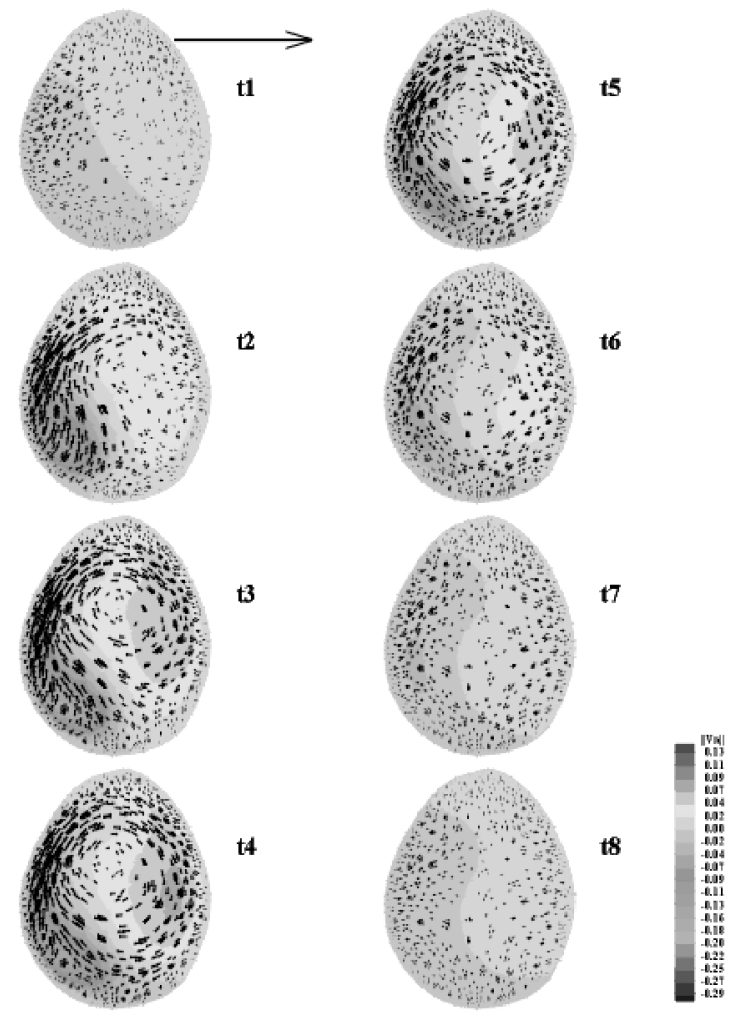

Figure 4b. Evolution of the inflow-outflow patterns and cross-flow vectors for the cross-section 'b' of the first aneurysm. (Original in colour)

same section (a), at the neck of the first sack). The maximum difference in location between the finest grid and our reference grid is less than 5\% and it is very close to the solid wall, where the velocity is of small magnitude. On the average, the spatial difference between this moderately fine (and clinically feasible in terms of necessary computer time requirements) grid and the finest grid tested is around $1 \%$, all errors non-dimensionalized with the aneurysm neck diameter. For the rest of the present study, a grid of 483,871 elements, with a second order discretization scheme in space and a second order Crank-Nicholson scheme for the temporal discretization was used.

Several computations were performed, for conditions corresponding to various heartbeat rates and blood flow rates, but for the results presented herewith the mean Reynolds number (based on the mean velocity and the vessel diameter at the entrance), was set equal to 300 . We should note however that although the details of the computed flow fields were of course influenced by the exact features of the pulsatile forcing, the trends observed were consistent, and so were the main conclusions drawn from these computations. An idealized pulsatile waveform was used as the inlet boundary condition, in the same spirit as in [12]. Figure 3 de- 


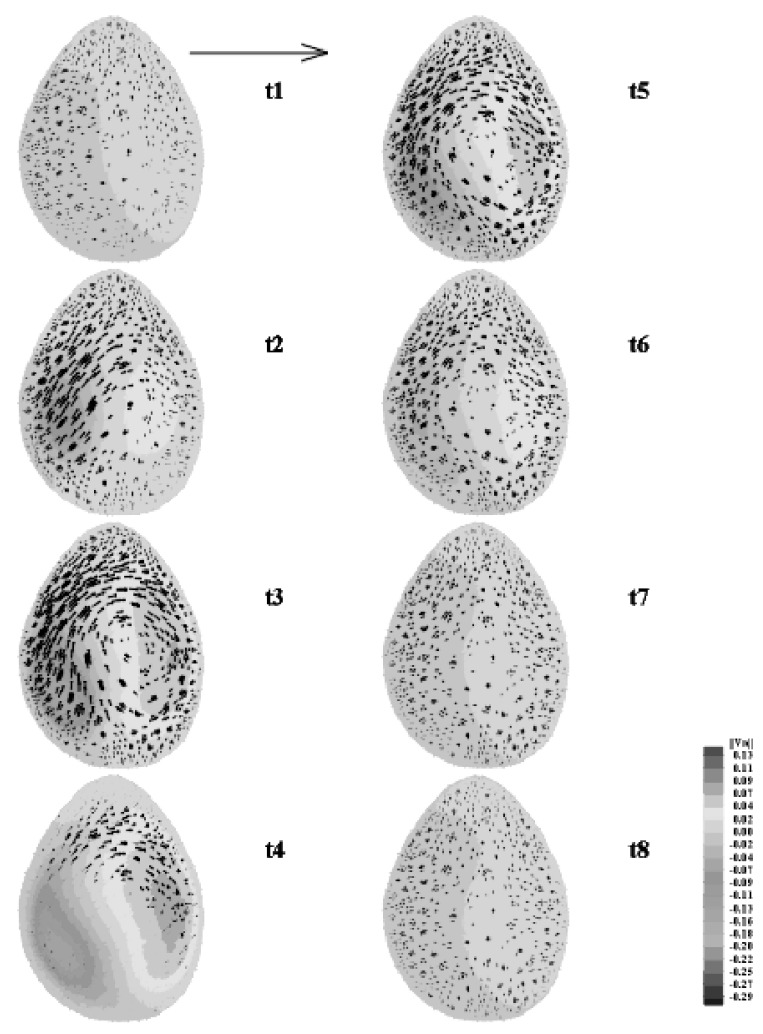

Figure 4c. Evolution of the inflow-outflow patterns and cross-flow vectors for the cross-section 'c' of the first aneurysm. (Original in colour)

picts the waveform used in terms of instantaneous over mean Reynolds number vs. time normalized with the heartbeat. The Womersley number, $a=R \sqrt{\omega / v}$ (where $R$ is an average radius, $\omega$ is the angular frequency and $v$ the kinematic viscosity of blood) was set equal to 4.8. The mean inlet velocity was used for the non-dimensionalization of all velocities. A fixed pressure boundary condition was specified for both distal outflow boundaries. The dimensionless computational time step was 0.01 , normalized with the heartbeat period. For the cases simulated in this study, certain simplifications were made, like assumptions of non-compliant walls and constant viscosity.

\section{Results and Discussion}

Figure 1 depicts a shaded view of the computational domain, characteristic dimensions along with the designation of the aneurysm names and cross-sections, used throughout this study. We shall name the aneurysm that is closer to the inflow boundary 'first aneurysm'. Moreover, we shall utilize the letters 'a', 'b', 'c' and 'd' for the four cross-sections of each aneurysm, starting from the aneurysm neck (slice 

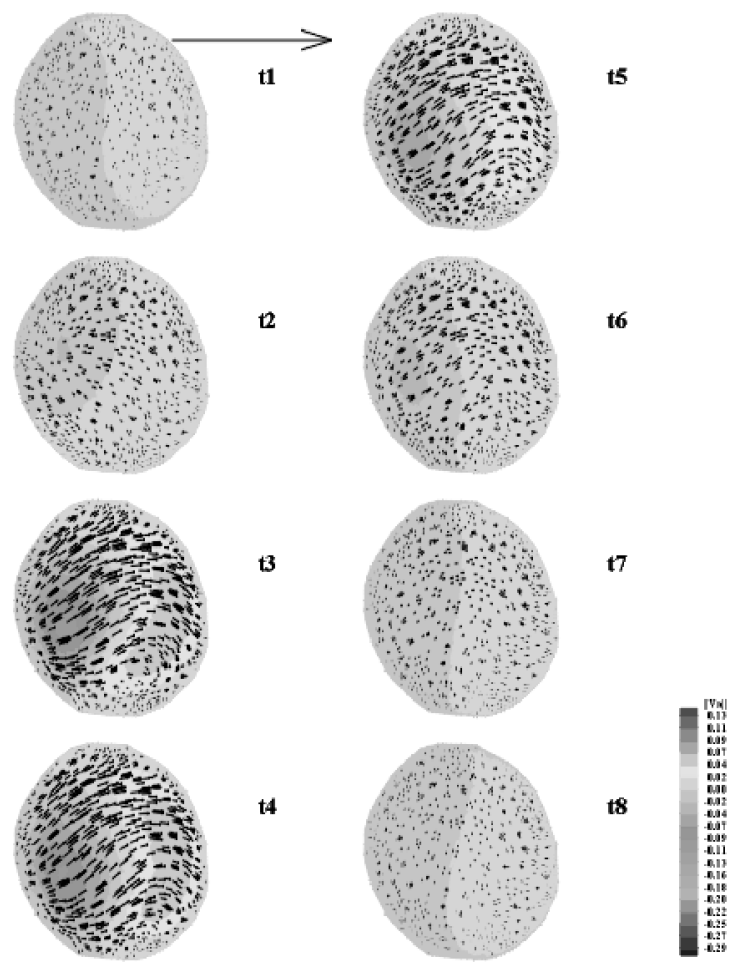

Figure 4d. Evolution of the inflow-outflow patterns and cross-flow vectors for the cross-section ' $d$ ' of the first aneurysm. (Original in colour)

'a') and going towards the aneurysm fundus (slice 'd'). The time instants where results will be presented are as follows: $t_{1}=0.00, t_{2}=0.13, t_{3}=0.26, t_{4}=0.39$, $t_{6}=0.52, t_{6}=0.65, t_{7}=0.78$ and $t_{8}=0.91$, a convention used throughout this study. As mentioned before, the basic period (i.e. the cardiac cycle) is one time unit long, and all times mentioned are non-dimensionalized with respect to this time.

We have computed a total of five cardiac cycles and we observed no difference (i.e. full periodicity) after the second cycle. The results presented correspond to the last cycle simulated and are thus clearly past all initial transients. Figures $4 \mathrm{a}$, $4 \mathrm{~b}, 4 \mathrm{c}$ and $4 \mathrm{~d}$ show contours of the normal velocity vector for the four control sections of the first aneurysm. Moreover, cross-flow vectors are depicted, showing the instantaneous secondary motion patterns. We can readily observe that significant secondary motion prevails, for section ' $a$ ' in particular, that is damped as we move towards the fundus (section ' $d$ '). Moreover, the inflow-outflow patterns observed oscillate significantly, with an actual switching of the inflow and the outflow areas been very apparent in the first section. Whereas we see a consistent secondary motion (clockwise in the figures) for sections located in the middle of this first aneurysm, this motion is much more complex on the entry section, but also at the fundus section. 


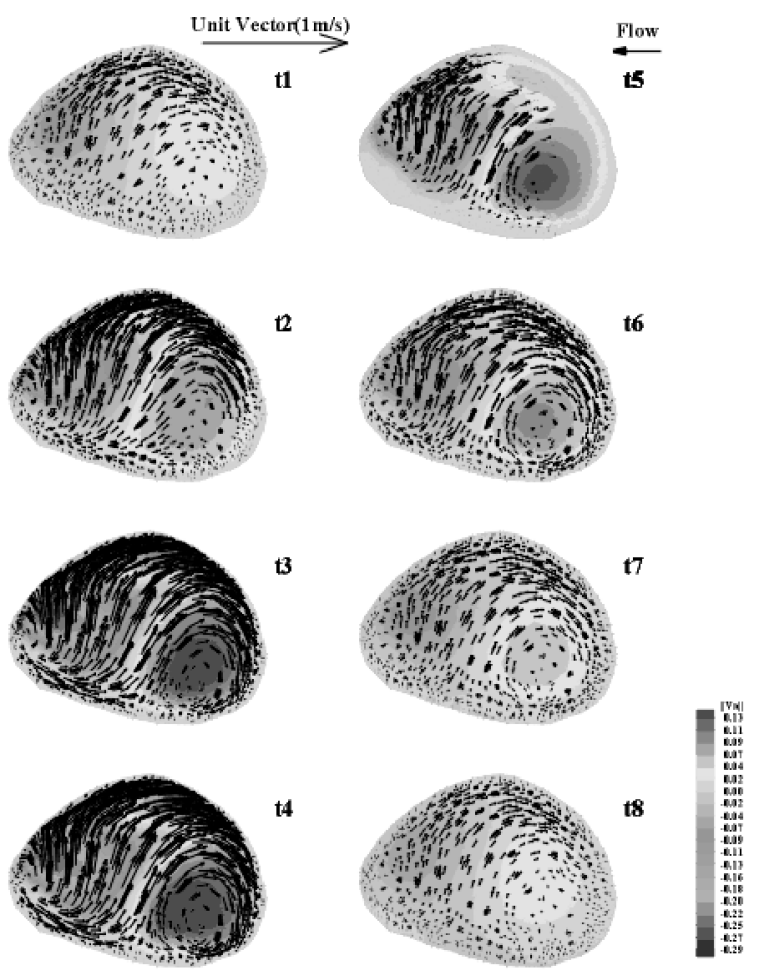

Figure 5a. Evolution of the inflow-outflow patterns and cross-flow vectors for the cross-section ' $a$ ' of the second aneurysm. (Original in colour)

The situation changes significantly when similar observations are made for the second aneurysm. Here, a remarkable insensitivity towards the strong pulsatility of the inlet flow is observed, with regard to the entrance cross-sectional areas where inflow and outflow is occurring. As we can see in Figures 5a, 5b, 5c and 5d, the intensity of the secondary motion is also very significant, but the inflow and outflow regions are more or less fixed in space and occupy approximately the same region on the aneurysm neck section for all time instants depicted. With respect to this parameter (inflow/outflow regimes), that is of interest in the present work, the fluid that feeds the second aneurysm emanates from such a subset of the cross-section between the first and second aneurysm where the pulsatility is not as strong as that of the inflow section. We shall revisit this issue in the sequel.

The cross-flow velocity patterns in this second aneurysm seem to be significantly more organized and consistent, for all the control sections. Actually, as we can see from these figures, there seem to be two very distinct, counter-rotating structures, that coincide more or less with the inflow-outflow patterns of all sections, except the very last one (Figure 5d) where the influence of the second aneurysm fundus wall damps the secondary motion significantly. Moreover, the geometry of this second sac, departing drastically from the more or less oblate 


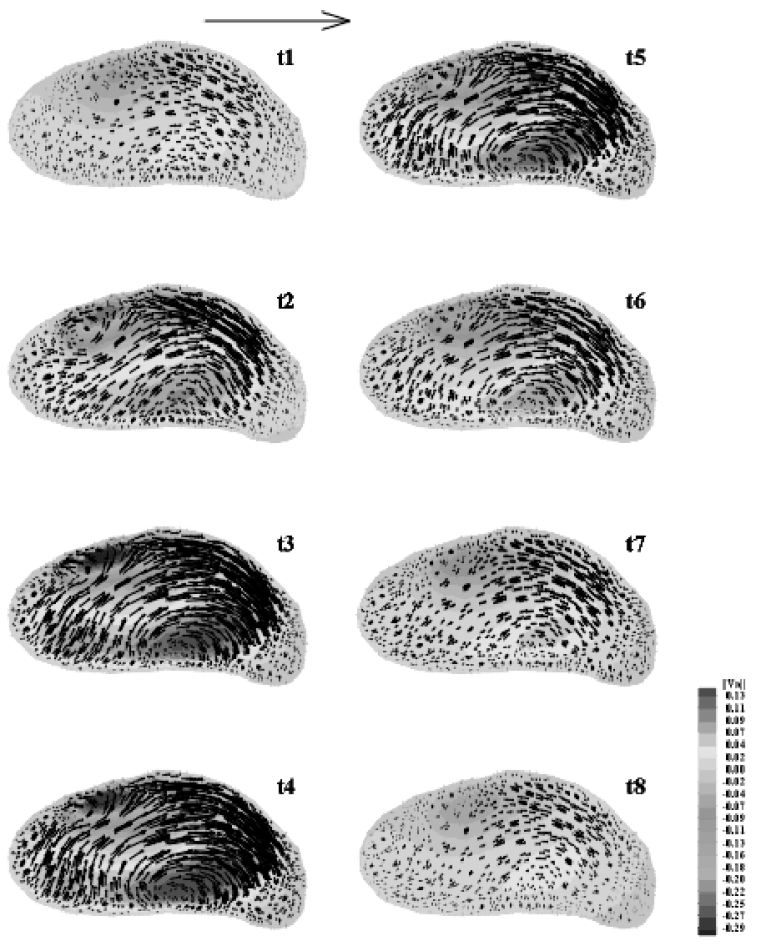

Figure 5b. Evolution of the inflow-outflow patterns and cross-flow vectors for the cross-section ' $b$ ' of the second aneurysm. (Original in colour)

spheroid we see at the first aneurysm, affects the emerging flow patterns, something that is quite obvious in Figure $5 b$.

The profoundly different nature of the flow in the two aneurysms can possibly be attributed to the fundamentally different locations and orientations they occupy with respect to the parent vessel: As we can see in Figure 1, the first aneurysm neck is oriented almost parallelly to the mean flow. In this respect, this aneurysm is acting like a cavity, driven by the oscillatory flow, and at the same time damping through the intense secondary motion it is developing some of the pulsatility that is convected downstream. Contrarily to this, the second aneurysm is located almost at a direct impingement angle with respect to the parent vessel. The main argument that can be made at this point is that the blood flow particulars for the cerebral vasculature pathology studied are not only patient-specific, something that has been identified for quite some time now and has diverted the computational efforts of researchers from generic idealized geometries to image-guided configurations, but there is also great differentiation for the same patient. Thus, for medical intervention planning procedures to be effective, it is obvious that the maximum possible amount of patient-specific information must be taken into account. 

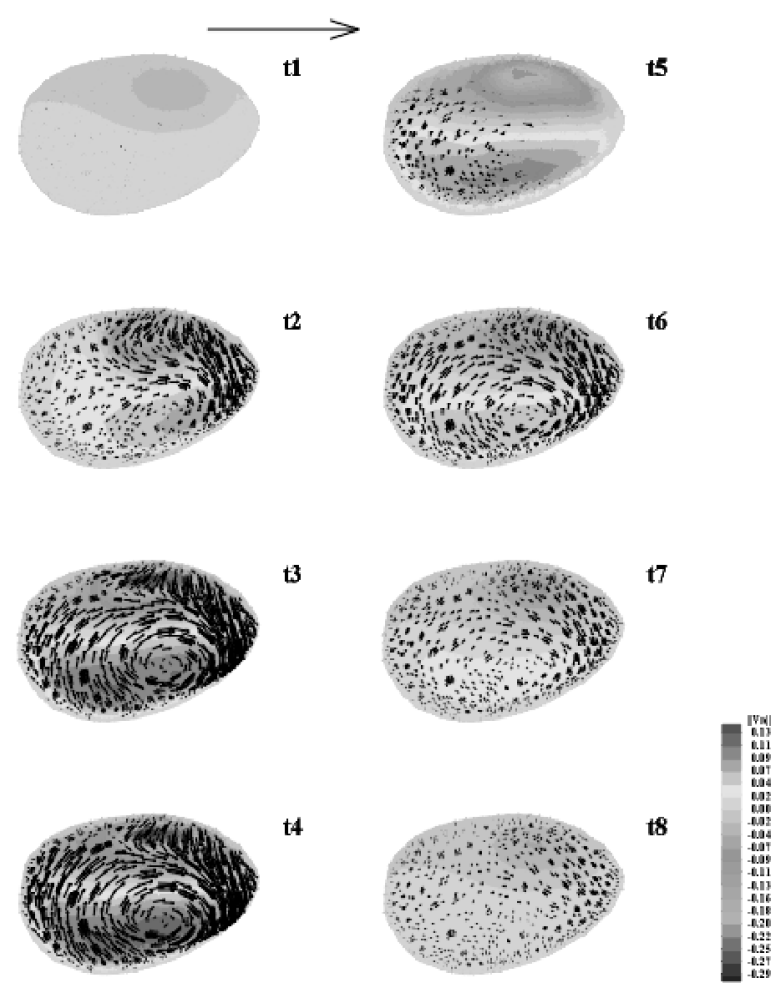

Figure 5c. Evolution of the inflow-outflow patterns and cross-flow vectors for the cross-section 'c' of the second aneurysm. (Original in colour)

A closing remark can be made at this stage in connection with the principal objective of this work which is the identification of the efficacy of CFD in general and computational haemodynamics in particular to answer practical questions with respect to optimal coil embolization procedures for cerebral saccular aneurysms. There is now convincing evidence, originating from large scale clinical randomized trials [21], that the preferred approach for cerebral aneurysm treatment is indeed coil embolization. Therefore, the need to plan and optimize this technique emerges more dominant than ever. This approach is based on the effort to exclude the sac from the blood circulation by establishing a stable, coil-reinforced, clot in the fundus. Thus, one predominant concern when this treatment is applied is connected with the basic assumption, i.e. that the aneurysm sac is indeed isolated from the flowing blood, especially for partially occluded aneurysms. We feel that there is currently no rigorous way that this question can be answered, and that computational simulation offers, possibly, the only viable alternative. 

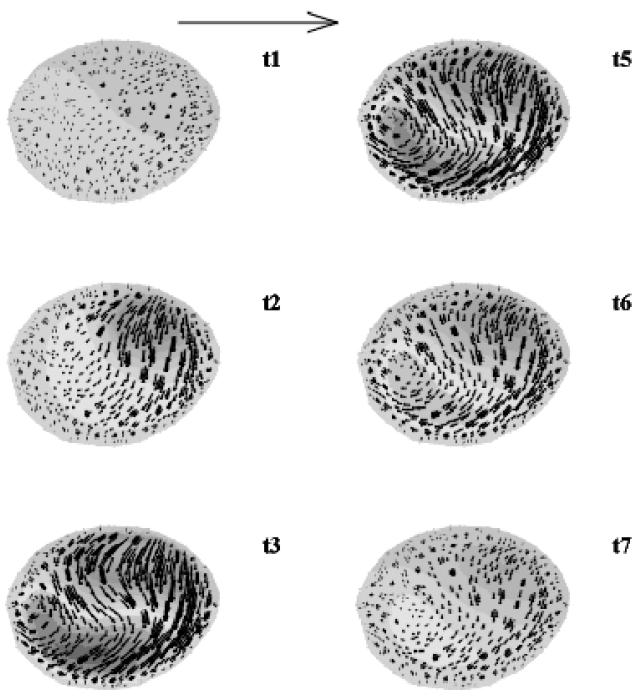

t3
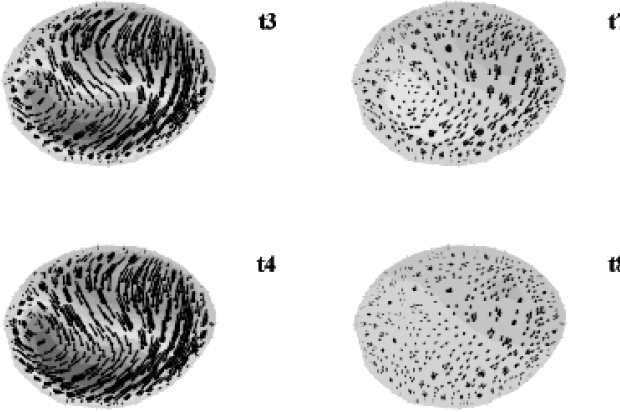

t4
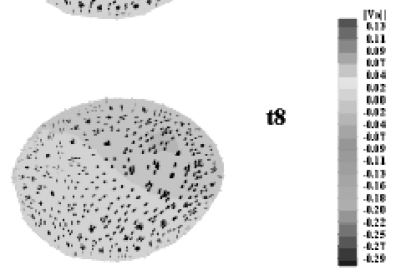

Figure 5d. Evolution of the inflow-outflow patterns and cross-flow vectors for the cross-section 'd' of the second aneurysm. (Original in colour)

\section{Conclusions}

In the present work, we have examined the haemodynamics of the pulsatile flow of a patient's right internal carotid artery, exhibiting two saccular aneurysms. The principal finding of this work is that the two aneurysms respond to the flow pulsatility in profoundly different ways: the first aneurysm, being oriented like a driven cavity, is strongly affected by the unsteady nature of the flow and at the same time damps a significant amount of this unsteadiness, before it reaches the second sac. This second aneurysm experiences a direct impingement of the damped flow and shows a remarkable insensitivity to the pulsatile flow patterns. The present study is driven by the need to establish a technique that can identify the optimum regions for coil embolization. Based on the observations made, we conclude that this identification can benefit greatly from simulations of the nature performed herewith. Such optimum embolization regions seem to be strongly connected not only with patient-specific but also with aneurysm-specific haemodynamics and thus insight on this kind of individual-based blood flow data can prove valuable for advanced intervention techniques. 


\section{Acknowledgements}

We would like to thank: Professor E. Hoffman (University of Iowa) for sharing the CT data used in this study with us; Professor D.A. Steinman (John P. Robarts Research Institute \& University of Western Ontario) for providing his expertise in the surface reconstruction of the geometry used and for insightful discussions; CFD Research Corporation (Huntsville AL) for allowing the use of their software suite; Dr. S. Kollias, Dr. P. Summers and Professor A. Valavanis (Neuroradiology Institute, University Hospital Zürich) for insightful discussions. The financial support of the ETH Forschungskommission is kindly acknowledged.

\section{References}

1. Bannermann, R.M., Ingall, R.M. and Graf, C.J., The familial occurrence of intracranial aneurysms. Neurology 20 (1970) 283-292.

2. Böcker, W., Denk, H. and Heitz, Ph.U., Pathologie, Urban \& Schwarzenberg (1997).

3. Butty, V.D., Gudjonsson, K., Buchel, P., Makhijani, V.B., Ventikos, Y. and Poulikakos, D., Residence times and basins of attraction for a realistic right internal carotid artery with two aneurysms. Biorheology 239 (2002) 387-393.

4. Chatziprodromou, I., Ventikos, Y. and Poulikakos, D., Numerical prerequisites for the utilization of computational haemodynamics in medical intervention planning: Application on cerebral vasculature pathologies. (2003) submitted.

5. Gill, J.D., Ladak, H.M., Steinman, D.A. and Fenster, A., Accuracy and variability assessment of a semi-automatic technique for segmentation of the carotid arteries from 3D ultrasound images. Medical Physics 27 (2000) 1333-1342.

6. Hoffman, E., Private communication (2001).

7. Johansen, K.H., Aneurysms. Scientific American 247 (1982) 110-125.

8. Kassell, N.F. and Torner, J.C., Epidemiology of intracranial aneurysms. Internat. Anesthesiol. Clin. 20 (1982) 13-17.

9. Löw, M., Perktold, K. and Raunig, R., Hemodynamics in rigid and distensible saccular aneurysms: A numerical study of pulsatile flow characteristics. Biorheology 30 (1993) 287-298.

10. Makhijani, V.B., Yang, H.Q., Dionne, P.J. and Thubrikar, M.J., Three-dimensional coupled fluid-structure simulation of pericardial bioprosthetic aortic valve function. ASAIO J. 43 (1997) 387-391.

11. Mumenthaler, M. and Mattle, H., Neurologie. Thieme (1997).

12. Myers, J.G., Moore, J.A., Ojha, M., Johnston, K.W. and Ethier, C.R., Factors influencing blood flow patterns in the human right coronary artery. Ann. Biomed. Engrg. 29 (2001) 109-120.

13. Perktold, K., On the paths of fluid particles in an axisymmetrical aneurysm. J. Biomech. 20 (1987) 311-317.

14. Prakash, S. and Ethier, C.R., Requirements of mesh resolution in 3D computational hemodynamics. J. Biomech. Engrg. Trans. ASME 123 (2001) 134-144.

15. Reininger, A.J., Reininger, C.B., Heinzmann, U. and Wurzinger, L.J., Residence time in niches of stagnant flow determines fibrin clot formation in an arterial branching model - Detailed flow-analysis and experimental results. Thrombosis and Haemostasis 74 (1995) 916-922.

16. Rinkel, G.J.E., Djibuti, M., Algra, A. and van Gijn, J., Prevalence and risk of rupture of intracranial aneurysms. A systematic review. Stroke 29 (1998) 251-256.

17. Steiger, H.-J., Pathophysiology of Development and Rupture of Cerebral Aneurysms. Acta Neurochirurgica Supplementum, Vol. 48, Springer, Wien (1990). 
18. Steinman, D.A., Private communication (2001).

19. Sukumar, R., Athavale, M.M., Makhijani, V.B. and Przekwas, A.J., Application of computational fluid dynamics techniques to blood pumps. Artificial Organs 20 (1996) 529-533.

20. Weir, B., Aneurysms Affecting the Nervous System. Williams and Wilkins, Baltimore, MD (1987).

21. International Subarachnoid Aneurysm Trial (ISAT) Collaborative Group, International subarachnoid aneurysm trial (ISAT) of neurosurgical clipping versus endovascular coiling in 2143 patients with ruptured intracranial aneurysms: A randomized trial. The Lancet 360 (2002) 1267-1274. 